\title{
Characteristics of epiphytic microorganisms of wheat plants antagonists of phytopathogenic fungi
} Aldosari M.D., Ksenofontova O.Y.

Saratov national research state University named after N. G. Chernyshevsky, Saratov, Russia E-mail: ksenofontova64@mail.ru

Key message. Epiphytic microbiological complex of the surface of spring wheat plants of the Saratov 70 variety was studied. Among of the selected epiphyte cultures, strains of fungicide producers were screened for the genera Alternaria, Aspergillus and Fusarium.

Keywords: Epiphytic microorganisms, antagonists, phytopathogenic fungi, Bacillus, Alternaria, Aspergillus, Fusarium

Epiphytic microflora of plants plays an important role in the life of plants, namely, it provides protection of plants from pathogens [1]. In nowaday, the damage to agricultural plants by phytopathogenic microorganisms is estimated at $12 \%$. It was found that the most of the plant pathogens are fungi [2]. Chemical protection of plants from phytopathogens still occupies a leading place in the arsenal of control measures. However, it is not environmentally safe and must be combined with biological means of protection. The use of various biological preparations for the control of phytopathogens is one of the promising methods of biological protection of plants from mycoses [3]. As biological control measures against phytopathogenic fungi, the most practical interest is represented by antagonistic microorganisms, the search for which is advisable to conduct among the microbial population of plants.

All of the above has determined the purpose of our research to study the antagonistic activity of wheat plant phylloplan microorganisms in relation to phytopathogenic fungi. Our experiments were aimed at studying the epiphytic microbiological complex of the surface of spring wheat plants of the Saratov 70 variety. The composition of epiphytic microflora was studied at various stages of plant development: germination, tillering, earing and maturation. Among the selected epiphyte cultures, strains of fungicide producers were screened for the genera Alternaria, Aspergillus and Fusarium.

Methods. For the work, a pilot site was selected in the fields of the research in Agricultural Research Institute of South-East Region in Saratov, where all the studied plants were kept in identical conditions. Sampling for the study of microoragnisms of phylloplanes was carried out by the method of imprinting the upper and lower leaf blade. For research, 30 plants were selected at a certain stage of development. Cultivation of microorganisms was carried out at $28 \pm 2^{\circ} \mathrm{C}$ for $2-5$ days on a potato medium and potato-dextrose agar. Isolated cultures of bacteria and fungi were identified using standard methods. The antagonism was determined using agar blocks on potato-dextrose agar.

Results. The analysis of the obtained results allowed us to establish that the most common bacteria were microorganisms of the genera Bacillus, Paenibacillus, Lactobacillus, Kurthia, Arthrobacter, Rhodococcus, Sarcina, Pseudomonas, Pantoae, Erwinia, Escherichia. The dependence of the number of microorganisms of the phase of wheat development was revealed. The largest number of epiphytic bacteria were isolated during the tillering and stemming phases. The most effective producers of fungicidal substances among epiphytes were bacteria of the genus Bacillus, which intensively suppressed the growth of fungi of the genera Alternaria and Aspergillus.

1. Selikhova A. A. Epiphytic microflora of plants as a specific factor of plant immunity. // Young scientist, 2019. No. 51 (289). P. 280-282.

2. Sanin S. S., Ibragimov, T. Z., Strizhekozin Y. A. Method of calculation of losses of the wheat crop from diseases //Protection and quarantine of plants. 2018 (1). P. 11-15.

3. Azizbekyan R. R. Biological preparations for the protection of agricultural plants / / Biotechnology. 2018.34 (5). P. 3747. 